\title{
EFEITOS DO CPPU E GA 3 NO CULTIVO DE UVA-'ITÁLIA' NA REGIÃO DO SUBMÉDIO SÃO FRANCISCO, NORDESTE DO BRASIL ${ }^{1}$
}

\author{
CARLOS AUGUSTO MENEZES FEITOSA²
}

\begin{abstract}
RESUMO - Na região do submédio do São Francisco, a uva-'Itália' destaca-se com 63,2\% da área total plantada. Atualmente, é a principal variedade para exportação. O objetivo principal deste trabalho foi avaliar o efeito das doses do CPPU (forchlorfenuron) e $\mathrm{GA}_{3}$, no aumento do tamanho da baga, que foram aplicadas por meio de pulverização dirigida sobre os cachos durante a fase de pegamento dos frutos, quando as bagas haviam atingido, aproximadamente, $8 \mathrm{~mm}$ de diâmetro. Este experimento foi conduzido na Fazenda Cooperyama, no município de Juazeiro-BA. O delineamento experimental foi o inteiramente casualizado, com oito tratamentos e três repetições, avaliando-se 15 cachos por tratamento com as seguintes doses: 1 - Testemunha absoluta; $2-\mathrm{GA}_{3} 20 \mathrm{mg} / 1 ; 3-\mathrm{GA}_{3} 20 \mathrm{mg} / 1+$ CPPU $3 \mathrm{mg} / 1$; 4- GA $20 \mathrm{mg} / 1+$ CPPU $5 \mathrm{mg} / 1 ; 5-\mathrm{GA} 320 \mathrm{mg} / 1+$ CPPU $10 \mathrm{mg} / 1$; 6- CPPU $3 \mathrm{mg} / \mathrm{l}$; 7- CPPU $5 \mathrm{mg} / 1 ; 8$ - CPPU $10 \mathrm{mg} / 1$. O melhor resultado obtido no crescimento da baga deu-se com a aplicação do CPPU $10 \mathrm{mg} / 1$. Este tratamento diferiu significativamente da testemunha e da aplicação convencional com GA $20 \mathrm{mg} / 1$, aumentando $8 \%$ e 4,6\% o comprimento da baga, $13,6 \%$ e 7,3\% sua largura, e o peso de baga em $32 \%$ e $16,3 \%$, respectivamente. Nos tratamentos com CPPU aplicado isoladamente e/ou associado com $\mathrm{GA}_{3}$, a colheita foi retardada em oito dias. Não se verificou diferença significativa para os teores de sólidos solúveis; no entanto, foi constatado que a acidez titulável obteve valores mais elevados na testemunha e no tratamento com $\mathrm{GA}_{3}$, aplicando $20 \mathrm{mg} / \mathrm{l}$. Com relação ao peso do engaço, não foi verificada diferença estatística entre os tratamentos.
\end{abstract}

Termos para indexação: Vitis vinifera L., tamanho das bagas, reguladores de crescimento,CPPU, forchlorfenuron, $\mathrm{GA}_{3}$.

\section{EFFECT OF CPPU AND GA 3 IN GRAPES 'ITÁLIA' IN SUBMEDIUM SÃO FRANCISCO RIVER VALLEY REGION, NORTHEAST BRAZIL}

\begin{abstract}
The Itália grape cultivar participates with $63,2 \%$ of the total growing area in Submedium São Francisco River Valley it's the main variety for exportation. The main objective in this work was to evaluate the effect of CPPU (forchlorfenuron) and $\mathrm{GA}_{3}$ doses on the berry size increase. The products were applied by spraying them on the bunches during the fruit-set stage when the berry size was about $8 \mathrm{~mm}$ in diameter. The experiment was done in Cooperyama farm in Juazeiro-BA, Brazil. The experimental design was choosed at random with 8 treatments and 3 repetitions, evaluating 15 bunches per treatment with the following doses: 1 - Control; 2- $\mathrm{GA}_{3} 20 \mathrm{mg} /$

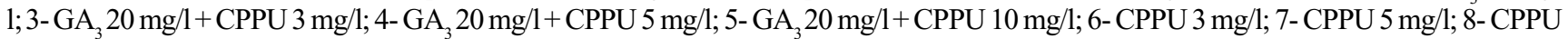
$10 \mathrm{mg} / 1$. The best result in the berry growth was with CPPU $10 \mathrm{mg} / 1$. This treatment differed significantly from the control and from $\mathrm{GA}_{3}$ $20 \mathrm{mg} / 1$ (the conventional treatment) increasing the berry length in $8 \%$ and $4,6 \% ; 13,6 \%$ and $7,3 \%$ its width and the berry weight in $32 \%$ and $16,3 \%$ respectively. In the treatments with CPPU applied alone and/or associated with $\mathrm{GA}_{3}$ the harvest was delayed in eight days. We could not find significative differences in soluble solids rates, but we found that the titrable acidity showed the highest values in the control and in the treatment with $\mathrm{GA}_{3} 20 \mathrm{mg} / \mathrm{l}$. About the stalks weigh we could not find statistical differences between the treatments.
\end{abstract}

Index terms: Vitis vinifera L., berries size, growth regulators, $\mathrm{CPPU}$, forchlorfenuron, $\mathrm{GA}_{3}$.

\section{INTRODUÇÃO}

A região do submédio São Francisco é considerada a maior região produtora de "uva fina de mesa" do País, com maior expressão no comércio internacional, respondendo também por $85 \%$ da exportação nacional.

A uva- 'Itália' (Piróvano 65) destaca-se como a mais importante uva para exportação do Vale do São Francisco.

$\mathrm{O}$ consumidor está cada vez mais exigente à procura de uvas com qualidade. Um aspecto importante é o tamanho da baga. Em conseqüência disso, surgiu a necessidade da realização de estudos em busca de novas alternativas. A utilização de $\mathrm{GA}_{3}$ na cultivar Itália é uma prática tradicional usada pelos pro- dutores, cuja dose varia de $15 \mathrm{mg} / 1$ a $30 \mathrm{mg} / \mathrm{l}$ aplicada em duas vezes, da seguinte forma: a primeira é aplicada aproximadamente aos 15 dias após a floração, ou quando a baga alcançar aproximadamente $8 \mathrm{~mm}$ de diâmetro. A segunda aplicação é realizada 15 dias após a primeira.

O CPPU (N-(2-cloro-4-piridil)-N'-feniluréia) é um regulador de crescimento que possui uma atividade fisiológica significante em diversas frutas, inclusive em uvas. O modo de ação é semelhante ao das citocininas (Dokoozlian et al., 1994) e, quando aplicado nos cachos após o pegamento dos frutos, o CPPU aumenta o tamanho das bagas e causa atraso à maturação.

Dependendo do estado de desenvolvimento da planta, da dose empregada, dos métodos de aplicação e de outras vari-

1 (Trabalho 054/2001). Recebido: 28/02/2001. Aceito para publicação: 25/01/2002. Trabalho apresentado no XVI Congresso Brasileiro de Fruticultura. Apoio Financeiro: WISER / VALEAGRO

2 Departamento Técnico da VALEXPORT, Caixa Postal 120, 56300-000, Petrolina, PE. E-mail: menefe@bol.com.br

Rev. Bras. Frutic., Jaboticabal - SP, v. 24, n. 2, p. 348-353, agosto 2002 
áveis, o CPPU pode produzir diferentes efeitos no cacho, tais como: favorecer a retenção de flores e aumentar o tamanho das bagas (Nickell, 1986, citado por Retamales et al., 1993).

Em aplicações de CPPU e $\mathrm{GA}_{3}$ na cultivar Thompson Seedless, observou-se um aumento de $28 \%$ no tamanho dos cachos em comparação às aplicações isoladas de GA 3 (Reynolds et al., citado por Schuck, 1994). Também foi verificado aumento no peso dos cachos com aplicações de GA 3 e CPPU.

Como este produto atua de forma localizada, é necessário que a aplicação seja uniforme em toda a superfície da fruta, pois a translocação do CPPU é aparentemente muito baixa (Morris et al., 1986; Tartarini et al., 1991; Neri et al., 1992; Biasi et al., 1992, citado por Intriere et al., 1992).

Um estudo realizado por Wolf et al., em 1994, mostrou resultados promissores no tamanho de bagas nas cultivares Sultanina, Flame Seedless e Muscat Seedless, melhorando a qualidade dos cachos nas três cultivares citadas, o que confirma a possibilidade de qualidade de exportação para as variedades estudadas com tratamentos de CPPU associado ao GA .

A aplicação de CPPU foi testada em diversas frutas como: uvas, kiwi, maçãs e pêras, obtendo um alto grau de eficiência nas colheitas (Nickell, 1986). A maioria das pesquisas com CPPU em uvas foram realizadas nos Estados Unidos e no Japão.

Segundo Pires (1998), dependendo do cultivo, o CPPU pode determinar aumento de espessura da ráquis e pedicelo, evitando assim excesso de desgrana de bagas.

Em vista disso, o presente trabalho teve como objetivo principal avaliar os efeitos da aplicação do CPPU (forchlorfenuron) em diferentes concentrações e aplicações isoladas e/ou combinados com o ácido giberélico, no diâmetro das bagas, no peso dos cachos, bem como na composição química dos frutos na cultivar Itália.

\section{MATERIAL E MÉTODOS}

O experimento foi instalado na Fazenda Cooperyama, no município de Juazeiro-BA, com coordenadas geográficas de 9³3' $\mathrm{S}$ e $40^{\circ} 36^{\prime} \mathrm{W}$ e altitude de $365 \mathrm{~m}$, com uma precipitação média anual de $400 \mathrm{~mm}$ e temperatura média de $26^{\circ} \mathrm{C}$.

A área experimental foi formada por plantas do cultivo da uva-'Itália' de 11 anos de idade, enxertada sobre o porta-enxerto IAC 572, espaçadas de 4 metros entre filas e 2,5 metros entre plantas, conduzidas no sistema de latada. Para todas as plantas, foram dispensados tratos culturais semelhantes.

O delineamento experimental foi o inteiramente casualizado, com 8 tratamentos e 3 repetições, considerando-se uma planta como repetição. O CPPU e GA foram aplicados com pulverizações dirigidas sobre os cachos, durante a fase de pegamento dos frutos, quando as bagas haviam atingido aproximadamente $8 \mathrm{~mm}$ de diâmetro. Os tratamentos com os reguladores de crescimento podem ser observados na Tabela 1.

Foram avaliadas as seguintes variáveis: peso dos cachos, diâmetro, comprimento e peso médio das bagas, peso do engaço, sólidos solúveis (SST), acidez total titulável (ATT) e relação SST/ ATT.

Através de amostras de 5 cachos por planta, avaliou-se o total de 15 cachos por tratamento, sendo que, para as avaliações de diâmetro e comprimento, peso médio das bagas e sólidos solúveis totais, foram analisadas 10 bagas por cacho coletado ao acaso. Para analisar a acidez total titulável, foram coletadas 100 bagas por tratamento, retirando-se uma amostra de $5 \mathrm{ml}$ do mosto, determinando-se a titulação com $\mathrm{NaOH} 0,1 \mathrm{~N}$. O teor de sólidos solúveis foi avaliado com refratômetro em autocompensação de temperatura.

Os resultados foram submetidos a uma análise estatística de variância, com a aplicação do teste de Tukey para comparação das médias.

\section{RESULTADOS E DISCUSSÃO}

\section{Crescimento das bagas}

O uso do CPPU, adicionado a doses usuais de ácido giberélico, produziu um incremento significativo no crescimento de bagas (Tabela 2). O maior tamanho no diâmetro e comprimento de bagas foi observado com o tratamento isolado de CPPU $10 \mathrm{mg} / \mathrm{l}$ e de uma segunda aplicação, aos 15 dias após, com $\mathrm{GA}_{3}$ na dose de $15 \mathrm{mg} / \mathrm{l}$ (Figuras 1 e 3), o que proporcionou um aumento de $8 \%$ no comprimento e $13,6 \%$ na largura das bagas, comparando-os com a testemunha. Nos demais tratamentos, não foram observadas diferenças significativas.

Este efeito foi observado no mesmo cultivo com a aplicação de CPPU 12 mg/l, aumentando em 19,4\% o diâmetro das bagas, comparado com a testemunha, trabalho realizado por Miele et al. (2000). O CPPU aplicado depois da floração na cv. Fujimino também aumentou o tamanho das bagas (Cai LiHong et al.,1996).

Neste experimento, as doses mais altas de CPPU, associadas ao $\mathrm{GA}_{3}$, não mostraram efeito sinérgico. Estes resultados não estão de acordo com os obtidos por outros autores (Wolf et al., 1994; Dokoozlian et al., 1994; Dias \& Maldonado, 1992; Retamales et al., 1993).

$\mathrm{Na}$ interpretação desses resultados acima, o sinergismo entre o CPPU, aplicado com a dose mais alta, associado com o $\mathrm{GA}_{3}$ (Tabela 1), obteve diâmetro de bagas menores. No entanto, a dose mais baixa de CPPU, nas mesmas condições citadas acima, mostrou-se um pouco mais eficaz, mas não superior à dose mais alta de CPPU aplicada isoladamente. Foi comprovado, em cultivares de uvas sem semente, como, por exemplo, nas cultivares Thompson seedless e Perlette, o sinergismo entre o CPPU e $\mathrm{GA}_{3}$; no entanto, no referido trabalho, a cultivar 'Itália' apresentou-se de maneira mais sensível a doses altas de CPPU, em conjunto com o $\mathrm{GA}_{3}$, comparando-a com as uvas sem sementes.

Em trabalhos realizados anteriormente, observou-se também resultado diferente com o uso das maiores doses testadas de CPPU (40 mg/l e $80 \mathrm{mg} / \mathrm{l}$ ), em conjunto com o GA, na cultivar Sultanina, apresentando um incremento no crescimento das bagas, tanto no comprimento como no diâmetro destas (Retamales et al., 1993).

Em virtude de um maior crescimento das bagas, obtevese um maior peso destas (Figura 2), considerando que o número de bagas não apresentou diferenças significativas.

O CPPU utilizado na dose de $10 \mathrm{mg} / \mathrm{l}$ promoveu um maior peso das bagas, obtendo um aumento de $32 \%$ e $16,3 \%$, diferindo estatisticamente da testemunha absoluta e do tratamento com $\mathrm{GA}_{3} 20 \mathrm{mg} / \mathrm{l}$, respectivamente. Os demais tratamentos não diferiram entre si de maneira significativa, com exceção da testemunha 
absoluta.

Resultados semelhantes foram observados na cultivar Thompson Seedless com a aplicação do CPPU na dose de $5 \mathrm{mg}$ / 1, um aumento de $40 \%$ no peso das bagas (Dokoozlian et al., 1994).

\section{Características do cacho}

Os tratamentos com CPPU mostraram uma tendência de aumento no peso dos cachos, aplicado isolado ou associado ao $\mathrm{GA}_{3}$, bem como o tratamento com $\mathrm{GA}_{3}$ aplicado isoladamente. No entanto, verificou-se o maior peso nos tratamentos com $\mathrm{GA}_{3}$ $20 \mathrm{mg} / \mathrm{l}+$ CPPU $5 \mathrm{mg} / \mathrm{l}$, CPPU $5 \mathrm{mg} / \mathrm{l} \mathrm{e} \mathrm{CPPU} 10 \mathrm{mg} / \mathrm{l}$.

Apesar disso, não foram notadas diferenças estatísticas entre os três tratamentos citados acima; no entanto, o melhor resultado foi com a aplicação do CPPU aplicado isolado, na dose de $10 \mathrm{mg} / \mathrm{l}$, aumentando o peso de cachos em 47,3\% e 11,4\%, comparando com a testemunha absoluta e com o GA, na dose de $20 \mathrm{mg} / 1$, respectivamente. Estes dados estão de acordo com os de Reynolds et al. (1992), citado por Schuck, no qual não foi observado nenhum efeito de sinergismo entre CPPU $+\mathrm{GA}_{3}$, em comparação a aplicações isoladas de $\mathrm{GA}_{3}$, em quatro cultivares de uva sem semente. Com relação ao peso do engaço, o CPPU, associado ao $\mathrm{GA}_{3}$ ou aplicado isolado, promoveu um maior engrossamento do engaço, mas não foi verificada diferença estatística entre os tratamentos.

O tratamento com CPPU $5 \mathrm{mg} / \mathrm{l}$ apresentou engaços com peso $31 \%$ superior ao da testemunha (Tabela 2 ). Resultados semelhantes foram encontrados no cultivo de uva-Perlette por Diaz \& Maldonado (1992), na dose de $10 \mathrm{mg} / \mathrm{l}$, obtendo um aumento de $88,2 \%$ comparado com a testemunha.

O CPPU, aplicado isolado ou em combinação com GA, resultou em cachos mais compactos, isto devido ao seu efeito positivo no diâmetro das bagas. $\mathrm{O}$ manejo usado para o raleio dos cachos foi realizado considerando a aplicação convencional de $\mathrm{GA}_{3} 20 \mathrm{mg} / \mathrm{l}$.

Observou-se, porém, que esta compactação dos cachos pode estar relacionada a um maior pegamento das bagas. Segundo Retamales et al. (1993), um maior crescimento das bagas induzido pelo CPPU causa uma maior compactação, impedindo um maior crescimento destas.

\section{Amadurecimento do fruto}

O CPPU, aplicado isolado ou combinado com o GA atrasou a acumulação de sólidos solúveis, pois a colheita foi retardada em 8 dias. As bagas tratadas com CPPU apresentaram uma coloração mais verde, comparando com a testemunha e a aplica-

TABELA 1 - Estágio fenológico, doses e datas de aplicações para os tratamentos na cultivar Itália. Juazeiro-BA, 2000.

\begin{tabular}{|c|c|c|}
\hline Tratamentos & $\begin{array}{c}\text { Bagas com } 8 \mathrm{~mm} \\
(05 / 02 / 2000)\end{array}$ & $\begin{array}{c}2 \text { semanas depois } \\
(20 / 02 / 2000)\end{array}$ \\
\hline $\begin{array}{l}\text { A } \\
\text { B } \\
\text { C } \\
\text { D } \\
\text { E } \\
\text { F } \\
\text { G } \\
\text { H }\end{array}$ & $\begin{array}{ccc} & 0 \\
& \mathrm{GA}_{3} 20 \mathrm{mg} / 1 \\
\mathrm{GA}_{3} 20 \mathrm{mg} / 1+\mathrm{CPPU} 3 \mathrm{mg} / 1 \\
\mathrm{GA}_{3} 20 \mathrm{mg} / 1+\mathrm{CPPU} 5 \mathrm{mg} / 1 \\
\mathrm{GA}_{3} 20 \mathrm{mg} / 1+\mathrm{CPPU} 10 \mathrm{mg} / 1 \\
\mathrm{CPPU} 3 \mathrm{mg} / 1 \\
\mathrm{CPPU} 5 \mathrm{mg} / 1 \\
\mathrm{CPPU} 10 \mathrm{mg} / 1\end{array}$ & $\begin{array}{lll} & 0 \\
\mathrm{GA}_{3} & 15 & \mathrm{mg} / 1 \\
\mathrm{GA}_{3} & 15 & \mathrm{mg} / 1 \\
\mathrm{GA}_{3} & 15 \mathrm{mg} / 1 \\
\mathrm{GA}_{3} & 15 \mathrm{mg} / 1 \\
\mathrm{GA}_{3} & 15 \mathrm{mg} / 1 \\
\mathrm{G} \mathrm{A}_{3} & 15 \mathrm{mg} / 1 \\
\mathrm{GA}_{3} & 15 \mathrm{mg} / 1 \\
\end{array}$ \\
\hline
\end{tabular}

TABELA 2 - Efeitos do CPPU e do ácido giberélico sobre o peso dos cachos, comprimento, diâmetro e peso de baga, peso do engaço, número de bagas, sólidos solúveis totais (SST), acidez total titulável (ATT) e relação SST/ATT de uvas cultivar Itália. Juazeiro-BA, 2000.

\begin{tabular}{|c|c|c|c|c|c|c|c|c|}
\hline & & & Bagas & & & & & \\
\hline Tratamentos & $\begin{array}{c}\text { Peso do } \\
\text { cacho } \\
\text { (g) }\end{array}$ & $\begin{array}{l}\text { Comprimento } \\
(\mathrm{mm})\end{array}$ & $\begin{array}{c}\text { Diâmetro } \\
(\mathrm{mm})\end{array}$ & $\begin{array}{c}\text { Peso } \\
(\mathrm{g})\end{array}$ & $\begin{array}{c}\text { Peso do engaço } \\
(\mathrm{g})\end{array}$ & $\begin{array}{c}\text { S S T } \\
{ }^{0} \text { Brix }\end{array}$ & $\begin{array}{l}\text { Acidez } \\
(\text { meq/1) }\end{array}$ & $\begin{array}{c}\text { Relação } \\
\text { SST/ATT }\end{array}$ \\
\hline $\begin{array}{l}\text { A- Testemunha } \\
\text { absoluta }\end{array}$ & $486,7 \mathrm{c}$ & $27,6 \mathrm{~d}$ & $22 \mathrm{~d}$ & $7,6 \mathrm{~d}$ & $13,9 \mathrm{a}$ & $16,2 \mathrm{a}$ & $118 \mathrm{a}$ & $18,3 \mathrm{e}$ \\
\hline B $-\mathrm{GA}_{3} 20 \mathrm{mg} / 1$ & $643,8 \mathrm{ab}$ & $28,5 \mathrm{~cd}$ & $23,3 \mathrm{c}$ & $8,6 \mathrm{c}$ & $17,2 \mathrm{a}$ & $15,4 \mathrm{ab}$ & $100 \mathrm{~b}$ & $20,5 \mathrm{~d}$ \\
\hline $\begin{array}{l}\text { C- } \mathrm{GA}_{3} 20 \mathrm{mg} / 1+ \\
\mathrm{CPPU} 3 \mathrm{mg} / 1\end{array}$ & $665,9 \mathrm{ab}$ & $29,7 \mathrm{ab}$ & $24,4 \mathrm{ab}$ & $9,7 \mathrm{ab}$ & $14,5 \mathrm{a}$ & $15,6 \mathrm{ab}$ & $76 \mathrm{~cd}$ & $27,3 \mathrm{~b}$ \\
\hline $\begin{array}{l}\text { D- } \mathrm{GA}_{3} 20 \mathrm{mg} / 1+ \\
\mathrm{CPPU} 5 \mathrm{mg} / 1\end{array}$ & $675 \mathrm{a}$ & $29,1 \mathrm{abc}$ & $24 \mathrm{bc}$ & $9,2 \mathrm{bc}$ & $16,2 \mathrm{a}$ & $15 \mathrm{~b}$ & $72 \mathrm{~d}$ & $27,8 \mathrm{~b}$ \\
\hline $\begin{array}{l}\mathbf{E}-\mathrm{GA}_{3} 20 \mathrm{mg} / 1+ \\
\mathrm{CPPU} 10 \mathrm{mg} / 1\end{array}$ & $523,8 \mathrm{bc}$ & $28,7 \mathrm{bcd}$ & $23,7 \mathrm{bc}$ & $8,9 \mathrm{c}$ & $17,5 \mathrm{a}$ & $15,6 \mathrm{ab}$ & $72 \mathrm{~d}$ & 28,8 a \\
\hline F- CPPU $3 \mathrm{mg} / 1$ & $621,9 \mathrm{abc}$ & $29,0 \mathrm{abc}$ & $23,7 \mathrm{bc}$ & $8,9 \mathrm{c}$ & $13,7 \mathrm{a}$ & $16,1 \mathrm{a}$ & $78 \mathrm{c}$ & $27,5 \mathrm{~b}$ \\
\hline G- CPPU $5 \mathrm{mg} / 1$ & 688,7 a & $29,3 \mathrm{abc}$ & $23,8 \mathrm{bc}$ & $9,1 \mathrm{bc}$ & $18,2 \mathrm{a}$ & $15,1 \mathrm{ab}$ & $74 \mathrm{~cd}$ & $27,2 \mathrm{~b}$ \\
\hline H- CPPU $10 \mathrm{mg} / 1$ & $717 \mathrm{a}$ & 29,8 a & $25 \mathrm{a}$ & $10 \mathrm{a}$ & $16,5 \mathrm{a}$ & $14,9 \mathrm{~b}$ & $76 \mathrm{~cd}$ & $26,2 \mathrm{c}$ \\
\hline F tratamento & $6,13 * *$ & $8,26 * *$ & $25,2 * *$ & $17,33 * *$ & $2,05 \mathrm{~ns}$ & $3,82 * *$ & $183,8 * *$ & $808,5 * *$ \\
\hline C.V \% & 20,27 & 3,27 & 2,92 & 7,72 & 29,06 & 5,89 & 3,31 & 1,19 \\
\hline
\end{tabular}

ns: não significativo

**: Significativo no nível de $1 \%$ probabilidade

Obs: Valores com letras iguais na coluna não diferem estatisticamente, ao nível de $5 \%$ de probabilidade, pelo teste de Tukey.

Rev. Bras. Frutic., Jaboticabal - SP, v. 24, n. 2, p. 348-353, agosto 2002 


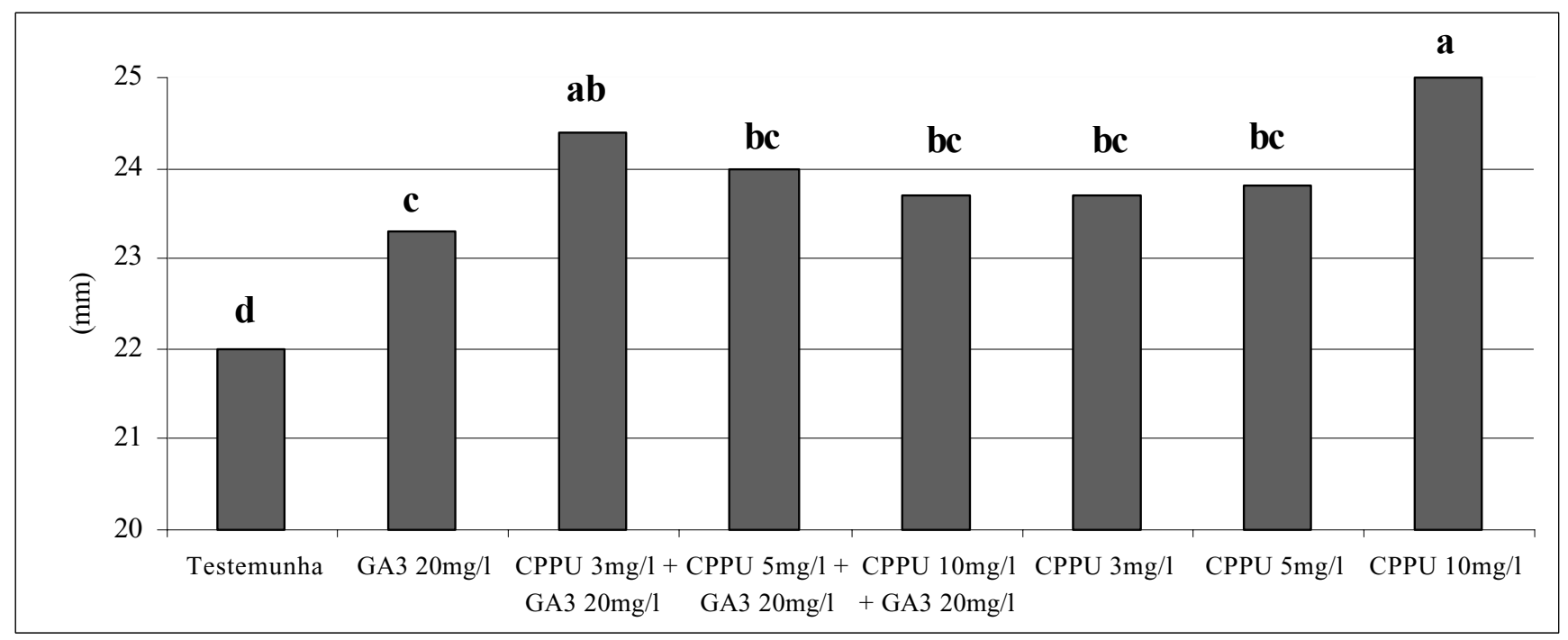

FIGURA 1 - Efeito do CPPU e GA 3 no diâmetro das bagas na cultivar Itália. Juazeiro-BA, 2000.

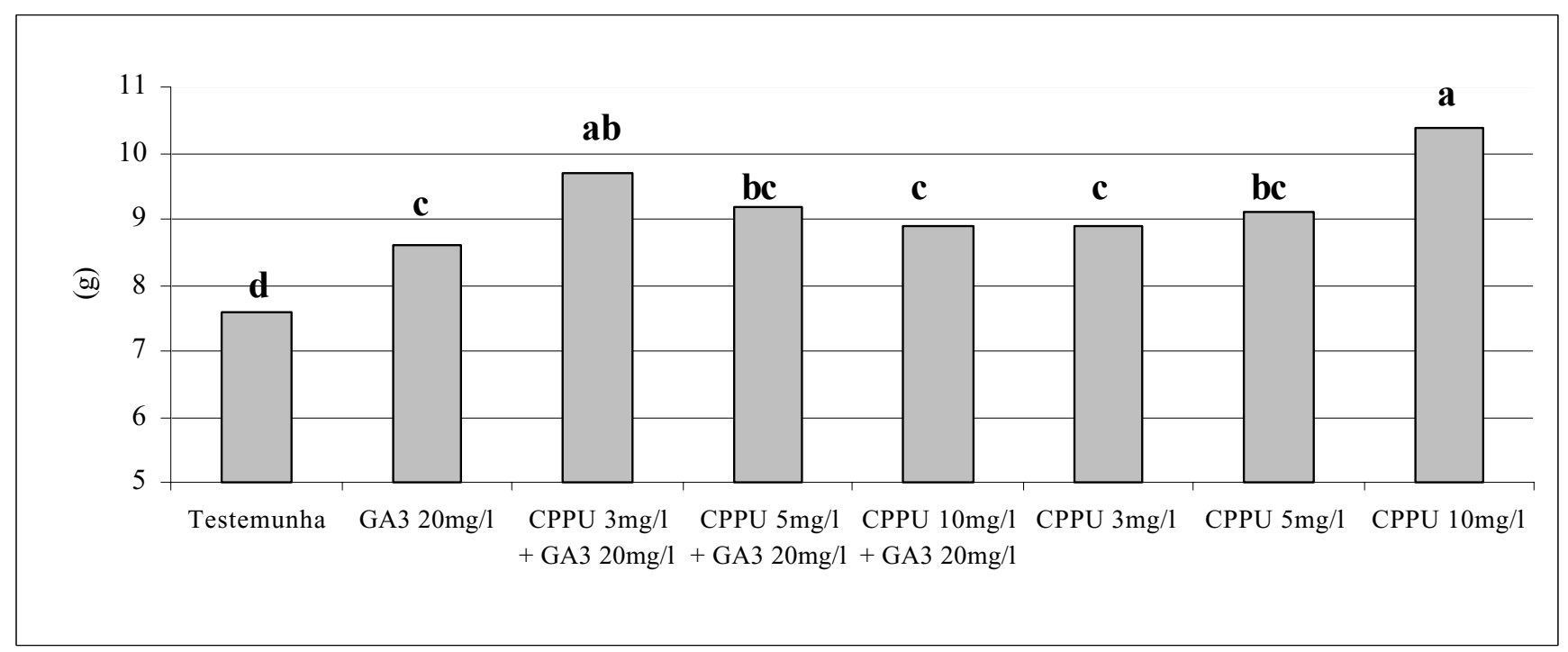

FIGURA 2 - Efeito do CPPU e GA, no peso das bagas na cultivar Itália. Juazeiro-BA, 2000.

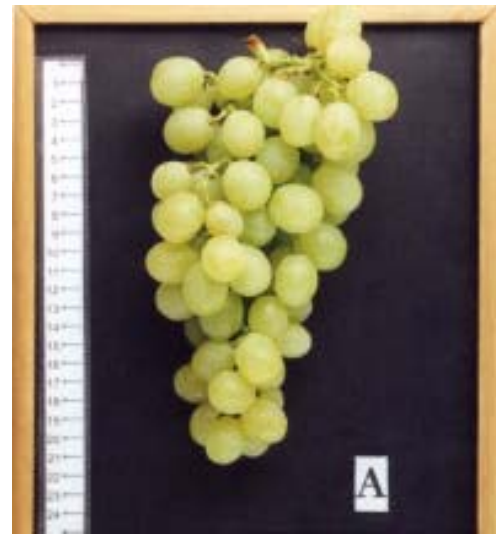

FIGURA 3 - Tratamento A: Testemunha absoluta

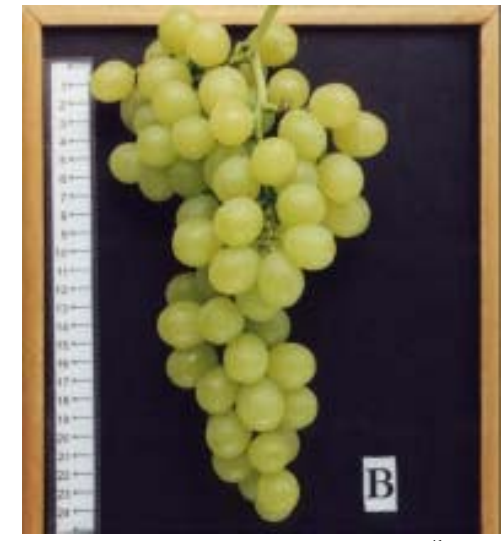

Tratamento B: $\mathrm{GA}_{3} 20 \mathrm{mg} / \mathrm{l}$ (Tratamento convencional)

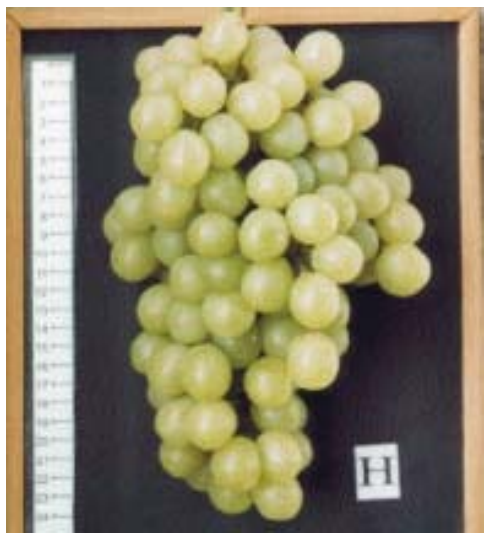

Tratamento H: CPPU $10 \mathrm{mg} / \mathrm{l}$ (Melhor resultado) 
ção isolada do $\mathrm{GA}_{3}$.

Segundo Thomas y Blakesley (1987), citado por Retamales et al. (1993), esses efeitos são esperados. É, pois, característica das citocininas prevenir a senescência e retardar a degradação da clorofila. Estes efeitos também foram observados por Reynolds et al. (1992); Intrieri et al. (1992); Nickell (1986).

Os frutos foram colhidos quando apresentavam um mínimo de $15^{\circ}$ Brix em todos os tratamentos. Os tratamentos A e F (Tabela 2) (respectivamente, testemunha, CPPU $3 \mathrm{mg} / \mathrm{l}$ ) diferiram estatisticamente dos tratamentos D e H $\left(\mathrm{GA}_{3} 20 \mathrm{mg} / \mathrm{l}+\mathrm{CPPU} 10\right.$ $\mathrm{mg} / \mathrm{l}$ e CPPU $10 \mathrm{mg} / \mathrm{l})$. Estes tratamentos tiveram os menores teores de sólidos solúveis devido a doses mais altas de CPPU.

Com relação à acidez total titulável, verificaram-se níveis mais altos de acidez nos tratamentos A e B (Testemunha, GA 20 $\mathrm{mg} / \mathrm{l}$ ), obtendo-se diferenças significativas com relação aos demais tratamentos (Tabela 2). Recente trabalho mostrou que o CPPU e o GA 3 , aplicados isolados ou em combinação, não apresentaram diferenças significativas comparados à testemunha no teor de acidez na uva-Perlette (Leão et al., 1999).

A relação brix/acidez mostrou diferenças significativas entre o tratamento E (CPPU $10 \mathrm{mg} / \mathrm{l}+\mathrm{GA}_{3} 20 \mathrm{mg} / \mathrm{l}$ ) e os tratamentos A e B (Testemunha e $\left.\mathrm{GA}_{3} 20 \mathrm{mg} / \mathrm{l}\right)$. Os últimos tratamentos citados tiveram uma relação brix/acidez mais baixa em comparação com os demais tratamentos (Tabela 2).

A colheita da uva-'Itália' tratada com CPPU, associada ao $\mathrm{GA}_{3}$, foi retardada em 8 dias, permitindo que os níveis de sólidos solúveis aumentassem nos tratamentos com CPPU, chegando a valores aproximados com a aplicação convencional de giberelina, permitindo também um decréscimo na acidez total. É característica do produto, determinar um atraso na acumulação de sólidos solúveis do fruto. Essa característica pode ser atribuída parcialmente, ao menos a um efeito de diluição, como conseqüência de um maior crescimento de bagas (Retamales et al., 1993).

\section{CONCLUSÕES}

Neste estudo, o efeito verificado do Forclorfenuron (CPPU), isolado e/ou associado ao ácido giberélico ( $\left.\mathrm{GA}_{3}\right)$, foi um crescimento adicional no tamanho das bagas na cultivar Itália.

Com base na análise e interpretação dos dados do experimento, permite-se destacar as seguintes conclusões:

1. O melhor resultado apresentado foi através da aplicação de CPPU $10 \mathrm{mg} / 1$, resultando em um incremento de $13,6 \%$ no diâmetro das bagas comparado com a testemunha. $\mathrm{O}$ peso das bagas também foi aumentado em $32 \%$.

2. O CPPU, associado ao $\mathrm{GA}_{3}$, retardou a colheita em 8 dias.

3. Com relação ao peso do engaço, não se obtiveram diferenças significativas entre os tratamentos.

Através dos resultados apresentados, pôde-se concluir que cachos de qualidade para exportação podem ser produzidos com tratamentos combinados de CPPU e GA.

No entanto, a utilização do CPPU, em áreas comerciais, requer a realização de ensaios a nível local.

\section{AGRADECIMENTOS}

O autor agradece ao Biólogo Gilberto José Nogueira e Silva, pelo apoio na realização do experimento, bem como aos técnicos da Valeagro, pela ajuda prestada na coleta de dados; ao Eng $^{\circ}$ Agr $^{\circ}$ Framber de Assis Bandeira Júnior - Gerente da Fazenda Cooperyama - , pela permissão do uso das áreas experimentais; ao Técnico Agrícola Romualdo Carlos Lemos Fonseca, pelo apoio operacional dado para a condução dos ensaios e à Ana Paula Costa Gomes, pela revisão da gramática e ortografia.

\section{REFERÊNCIAS BIBLIOGRÁFICAS}

LIHONG, C.; CHUGEN, H.; ZHENGRONG, L.; CAI, L. H.; HU, C. G.; LUO, Z.R. Study on the effect of several plant growth regulators on the grape berry size and quality. Department of Horticulture, Hoazhong Agricultural University, Wuhan, Hubei, China. South China Fruits, v. 25, n. 2, p.45-47, 1996.

DIAZ, H. D.; MALDONADO, L. A. Forchlorfenuron effects on berry size and maturity of Perlette and Flame seedless grapes. Proc. Plant Growth Reg. Soc. Am., v.19, p.123-128, 1992.

DOKOOZLIAN, N. K.; MORIYAMA, M.M.; EBISUDA, N. C. Forchlorfenuron (CPPU) increases the berry size and delays the maturity of Thompson Seedless table grapes. INTERNACIONAL SYMPOSIUM ON TABLE GRAPES PRODUCTION, 1994, Anaheim, California. Proceedings of the Davis: American Society for Enology and Viticulture/ University of California, 1994. p. 63-68.

INTRIERE, C.; FILIPPETTI, I.; PONI, S. The effect of N- (2-chloro4-pyridyl)-N'-phenylurea (CPPU) on berry growth and ripening of stenospermocarpic and seeded table grapes. Adv. Horticultural Science, 6 (1992): 137-143 Istituto di Coltivazione Arboree, Universitá di Bologna, 40126 Bologna, Italy.

LEÃO, P.C.S.; JUNIOR, E.C.L.; SANTOS, E.S. Efeitos do CPPU e ácido giberélico sobre o tamanho de bagas da uva-Perlette cultivada no Vale do São Francisco. Revista Brasileira de Fruticultura, Jaboticabal-SP, v. 21, n.1, p. 74-78, abril 1999.

MIELE, A.; DALL'AGNOL, I.; RIZZON, L.A. Efeito de reguladores de crescimento no tamanho da baga e na composição do mosto da uva-Itália. Revista Brasileira de Fruticultura, Jaboticabal-SP, v. 22, n. 2, p. 272-276, agosto 2000.

NICKELL, L. G. Effects of N- (2-chloro-4-pyridyl)-N'-phenylurea on grapes and other crops. Proc. Plant Growth Reg. Soc. Am., v.13, p.236-241, 1986.

PIRES, E.J. P. Emprego de reguladores de crescimento em viticultura tropical. Informe Agropecuário, Belo Horizonte, v.19, n.194, p.40-43, 1998. 
RETAMALES, J.; COOPER, T.; BANGERTH, F.; CALLEJAS, R. Efecto de aplicaciones de CPPU y GA $\mathrm{G}_{3}$ en el crecimiento y calidad de uva de mesa cv. Sultanina. Rev. Frutícola, vol. 14, n 3 Sep-Dic 1993.

REYNOLDS, A. G.; WARDLE, D. A.; ZUROWSKI, C.; LOONEY, N. E. Phenylureas CPPU and thidiazuron affect yield components, fruit composition, and storage potential of four seedless grapes selections. Journal of the American Society for Horticultural Science, Washington, v.117, n.1, p.85-89, 1992.
SCHUCK, E. Efeitos de reguladores de crescimento sobre o peso dos cachos, bagas e maturação da uva de mesa, cv. "Vênus". Revista Brasileira de Fruticultura, Cruz das Almas,v. 16, n.1, p. 295-306, 1994.

WOLF E, E.H.; VILJOEN, J.A.; NIEUWENHUYS, A.; LOUBSER, J.T. The effect of forchlorfenuron on bunch quality in table grapes. In: INTERNACIONAL SYMPOSIUM ON TABLE GRAPES PRODUCTION, 1994, Anaheim, California. Proceedings of the Davis: American Society for Enology and Viticulture/ University of California, 1994. p.50-53. 